\title{
Sleeping Beauty-Mediated Suicide Gene Therapy of Hepatocellular Carcinoma
}

\author{
Joon seok Song, ${ }^{1}$ Chul woo KIM, ${ }^{2}$ and Erin Rubin OCHOA ${ }^{1, \dagger}$ \\ ${ }^{1}$ University of Pittsburgh Medical Center, Division of Transplantation Pathology, Pittsburgh, PA 15213, USA \\ ${ }^{2}$ Seoul National University College of Medicine, Department of Pathology, Seoul 110-799, Korea
}

Received August 22, 2008; Accepted September 25, 2008; Online Publication, January 7, 2009

[doi:10.1271/bbb.80581]

The aim of this study was to use gene therapy via the Sleeping Beauty (SB) system to increase telomerase promoter activity to target hepatocellular carcinoma (HCC). In previous studies, we identified selective and increased expression of luciferase and suicide genes controlled by the hTERT (human telomerase reverse transcriptase) promoter and the SV40 enhancer in telomerase-positive cancer cell lines. Because telomerase is activated in about $80 \%$ of HCCs, it is likely that increasing the activity of the telomerase promoter with a suicide gene will effectively eradicate HCCs. We found that the telomerase promoter mediated SB system can efficiently insert transgene into HCC genomes. Also, telomerase promoter activity was increased using a SB vector expressing suicide gene HSV-TK (herpes simplex virus thymidine kinase) controlled by the hTERT promoter and a SV40 enhancer for the induction of telomerase-positive cancer-specific cell death. HCC cell lines transfected with pT.hTp.HSV-tk.Con with active helper plasmid and ganciclovir (GCV) significantly inhibited cancer cell growth. These results indicate that Sleeping Beauty transposon mediated suicide gene expression can be used in HCC-targeted cancer gene therapy.

Key words: Sleeping Beauty transposon; human telomerase reverse transcriptase (hTERT) promoter; SV40 enhancer; hepatocellular carcinoma; targeted cancer gene therapy

Genetic material can be introduced into chromosomes of all organisms via viruses or transposons. Sleeping Beauty (SB) transposon system, a type of mobile element that belongs to the $\mathrm{Tc} 1 /$ mariner class of transposons, is a promising tool for gene delivery due to its ability to express therapeutic genes stably as well as to infect resting cells. ${ }^{1,2)}$ At specific TA sequences, SB moves in a simple, cut-and-paste manner. Although popular tools of gene therapy, viral vectors are not ideal due to immunogenic problems, potential RCV (Replication Competent Virus) outbreaks, and difficulties in mass production. The advantages of the Sleeping Beauty transposon system include prolonged transgene expression without elicitation of an immunogenic response, no possibility of RCV, and ease of construction. SB is an excellent candidate for cancer gene therapy because it promotes long-lasting gene expression even in mitotically active tissues such as tumors, but also lacks many of the drawbacks related to viral vectors. ${ }^{3,4)}$
Telomerase is a ribonucleoprotein complex the function of which is to add telomeric repeats to chromosomal ends. It consists of two essential components, the telomerase RNA template (hTR) and the catalytic subunit (hTERT). The hTERT promoter is activated only in cells and tissues with telomerase activity, i.e., tumor or stem cells. ${ }^{5,6)}$ Because telomerase is activated in $80-90 \%$ of hepatocellular carcinomas (HCCs) ${ }^{7)}$ it is an ideal candidate for anti HCC cancer therapy. The aim of this study was to determine the effects of the HSV-TK gene under the control of the hTERT promoter with the SV40 enhancer delivered via $\mathrm{SB}$ on hepatocellular carcinogenesis. SB vector system which contains suicide gene HSV-TK controlled by the hTERTp and SV40 enhancer was constructed in order to suppress tumor cell growth and induce apoptosis in hepatocellular carcinoma cell lines. The results of this study indicate that the hTERT promoter and SV40 enhancer delivered via the SB are effective in targeted cancer gene therapy.

\section{Materials and Methods}

Cell culture. HepG2, Hep3B, and Huh7 (human hepatoma) cell lines were grown in DMEM (Gibco BRL, GmbH, Karlsrahe, Germany) supplemented with 10\% FCS (HyClone, Logan, UT), penicillin (50 units $/ \mathrm{ml})$, and streptomycin $(50 \mu \mathrm{g} / \mathrm{ml})$ in the presence of $5 \%$ $\mathrm{CO}_{2}$. Human normal primary hepatocytes (hNHeps) were purchased from Lonza (Lonza, Walkersville, MD) and maintained in hepatocyte culture medium (Lonza, Walkersville, MD). All cell lines except for normal human hepatocytes were obtained from the American Type Culture Collection (ATCC, Manassas, VA).

Construction of plasmids. The Sleeping Beauty-transposon system plasmids $^{1)}$ pT-MCS, pTnori, pCMV-SB, and pCMV-mSB (a transposase having a missense mutation in its C-terminal Asp-Asp-Glu (D, D(35)E) motif) were kindly provided by Dr. Mark A. Kay (Stanford University). In order to construct the transposon plasmid, pGL3-hTCon and pGL3-hT-TK-Con plasmids ${ }^{8)}$ were cut with KpnI/BamHI, a luciferase and the HSV-tk cassette were filled with DNA polymerase I and ligated with blunt-ended pT-MCS vector, resulting in pT.hTp.Con and pT.hTp.HSV-tk.Con vectors respectively (Fig. 1). pT.hTp.nori.Con was made from pTnori by inserting the human telomerase promoter and the SV40 enhancer. The SV40 promoter was replaced with hTERTp by cutting with $\operatorname{SexAI} / B g l \mathrm{II}$ in pTnori. The SV40 enhancer was inserted into the BsmI/BamHI sites of pTnori. The hTERT promoter and the SV40 enhancer region were amplified by PCR using Ex-Taq polymerase (Takara, Shiga, Japan) on the pGL3-hT-Con vector. The hTERTp F SexAI forward (5'-accaggtagtggattcgcgggcacaga- $\left.3^{\prime}\right)$, and hTERTp R $B g l I I$ reverse $\left(5^{\prime}\right.$-agatctagggcttcccacgtgcgcag- $\left.3^{\prime}\right)$ primers, and the SV40E F BsmI $\left(5^{\prime}\right.$-gcattcgatggagcgg- $\left.3^{\prime}\right)$, and SV40E R BamHI $\left(5^{\prime}-\right.$

$\dagger$ To whom correspondence should be addressed. Fax: +1-412-647-5237; E-mail: ochoaer@upmc.edu

Abbreviations: HCC, hepatocellular carcinoma; HSV-TK, herpes simplex virus thymidine kinase; GCV, ganciclovir; hTERT, human telomerase reverse transcriptase; SB, Sleeping Beauty transposon 


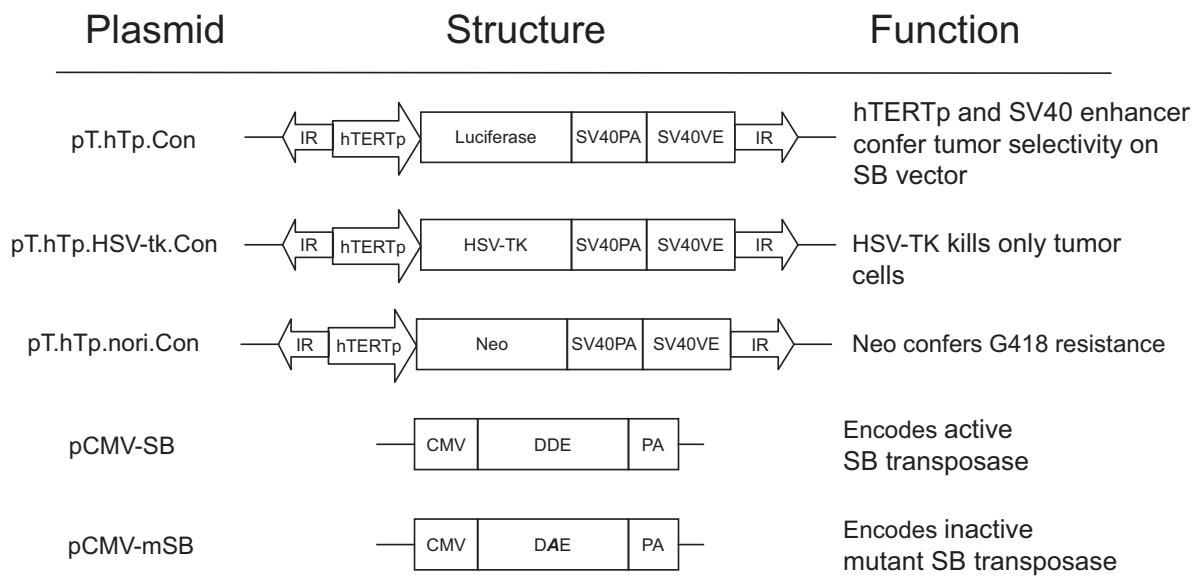

Fig. 1. Vectors for SB-Mediated Transposition.

ggatccgctgtggaatg- $\left.3^{\prime}\right)$ primers, both introducing restriction enzyme sites (underlined in the sequences) were used. PCR was carried out for 30 cycles at $98^{\circ} \mathrm{C}$ for $10 \mathrm{~s}$, and at $68^{\circ} \mathrm{C}$ for $1 \mathrm{~min}$.

Luciferase assay. Luciferase assays (Promega, Madison, WI) were performed according to the manufacturer's protocols using AutoLumat LB953 (Berthold, Wildbad, Germany). Briefly, $10^{5}$ cells seeded in a 35-mm tissue culture dish were exposed to a transfection mixture containing $2 \mu \mathrm{g}$ of luciferase reporter plasmids and $0.5 \mu \mathrm{g}$ of $\mathrm{pSV}-\beta$ galactosidase control plasmid vector (Promega, Madison, WI). $48 \mathrm{~h}$ after transfection, luciferase assays were performed according to the manufacturer's protocols and measured. The Simian virus 40 (SV40) promoter (pGL3-Promoter) was used as a positive control. The luciferase activity of the pGL3-Promoter plasmid in each cell line was considered to be 1 . A $\beta$-galactosidase assay was also performed with the same cell extracts to standardize for transfection efficiency. All of the data reported here were obtained from at least three independent experiments.

Transposition assay in cultured cells. To determine the transposition efficiency of the new Sleeping Beauty vector, we used a transient transfection assay. HepG2 cells $\left(5 \times 10^{5}\right)$ were seeded in 6-well dishes $24 \mathrm{~h}$ before transfection. pT.hTp.nori.Con $(1.5 \mu \mathrm{g})$ and pGL3-Control $(1.5 \mu \mathrm{g})$, pCMV-SB $(1.5 \mu \mathrm{g})$ or pCMV-mSB $(1.5 \mu \mathrm{g})$ were transfected with Lipofectamine 2000 (Invitrogen, Carlsbad, CA). Cells were cultured in DMEM containing G418 $(500 \mu \mathrm{g} / \mathrm{ml})$ for $10 \mathrm{~d}$. The relative transposition frequencies were determined by counting the number of G418-resistant $\left(\mathrm{G} 418^{\mathrm{R}}\right)$ foci on each plate after fixation in formaldehyde and staining with methylene blue.

Plasmid transfection with GCV treatment in vitro. HepG2, Hep3B, Huh7, and hNHeps cells $\left(10^{5}\right.$ cells each) were transfected with pT.hTp.HSV-TK.Con and pCMV-SB plasmid or pCMV-mSB using Lipofectamine 2000 (Invitrogen, Carlsbad, CA). After incubation at $37^{\circ} \mathrm{C}$ for $2 \mathrm{~d}, 2 \mathrm{ml}$ of media containing of $50 \mu \mathrm{g} / \mathrm{ml}$ concentration of Ganciclovir (GCV; InvivoGen, San Diego, CA) were added to the cells. All of the data shown in this report were obtained from at least three independent experiments.

ATP cell viability assay. ATP content was measured in accordance with the protocol of the CellTiter-Glo ${ }^{\circledR}$ Luminescent Cell Viability Assay kit (Promega, Madison, WI). Briefly, $100 \mu \mathrm{l}$ of assay reagent was added to the wells and mixed for $2 \mathrm{~min}$ at room temperature. After $10 \mathrm{~min}$, the intracellular ATP content was measured using an AutoLumat LB953 (Berthold, Wildbad, Germany).

\section{Results and Discussion}

To determine the variation in transcriptional activity of the hTERT promoter between HCC and normal cells, a transient transfection of luciferase reporter plasmid was performed (Fig. 2).

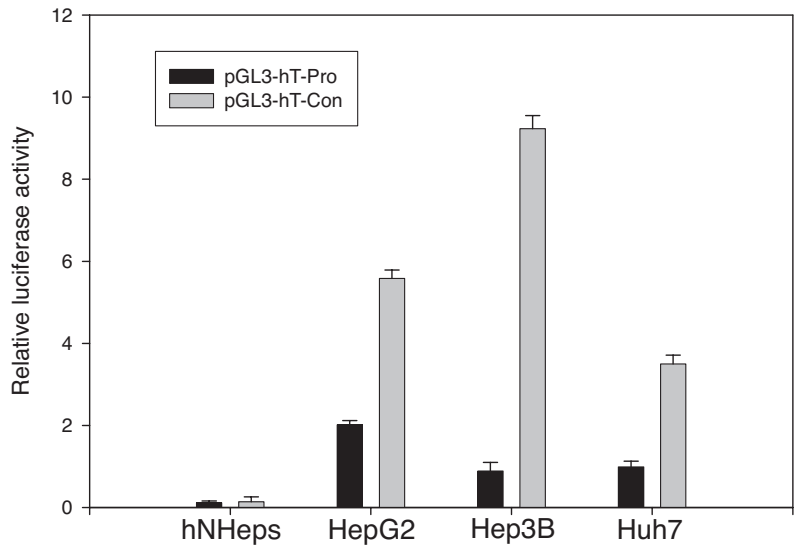

Fig. 2. Specific Expression of Luciferase Gene Imparted by the hTERT Promoter Plasmid Consisting in the hTERT Promoter with and without SV40 Enhancer.

Luciferase genes were introduced into primary hepatocytes (hNHeps) and HCC cell lines (HepG2, Hep3B, and Huh7). Relative luciferase activity was standardized by control plasmid pGL3Promoter transfection; bars, SD.

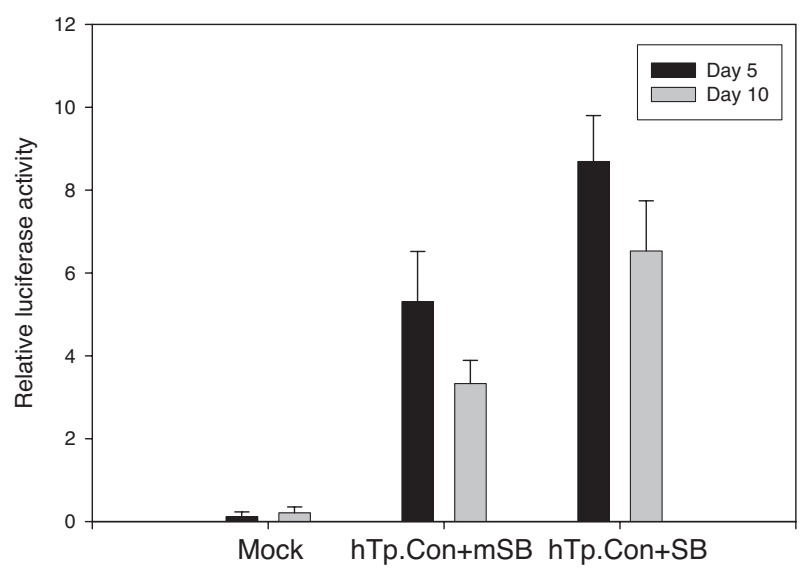

Fig. 3. Luciferase Assay for SB-Mediated Transgene Integration in Hep3B Cells Transfected with Donor Plasmid.

Mock, untransfected cells; hTp.Con, pT.hTp.Con; mSB, pCMV$\mathrm{mSB}$ (mutated SB transposase); SB, pCMV-SB (SB transposase).

Using the hTERTp promoter alone (pGL3-hT-Pro), luciferase expression increased 2-fold compared with the SV40 promoter in HepG2, but was almost same for Hep3B and Huh7 cells. Adding the SV40 enhancer (pGL3-hT-Con) increased hTERT promoter activity in HepG2, Hep3B, and Huh7 cells 2.7-, 10.3- and 3.5-folds 


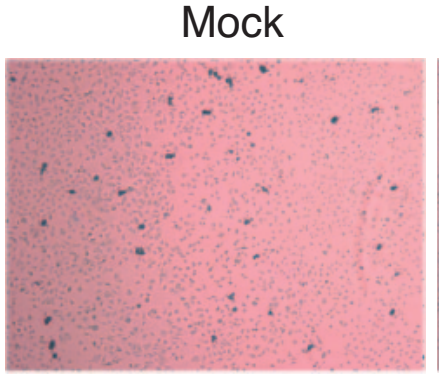

$42 \pm 5$
hTp.nori.Con+mSB

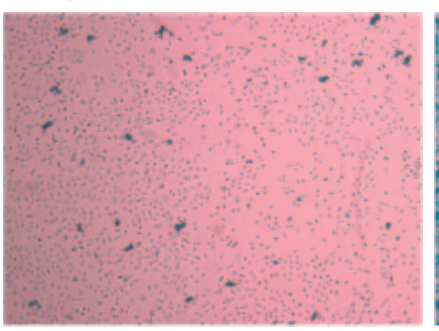

$45 \pm 4$
hTp.nori.Con+SB

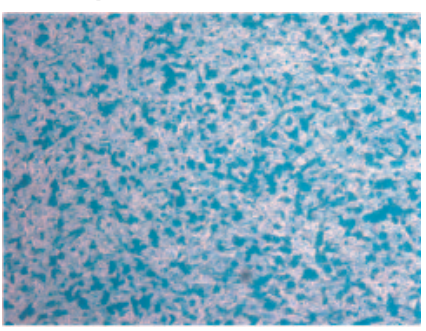

$2,256 \pm 52$

Fig. 4. Genetic Assay for SB-Mediated Transgene Integration in HepG2 Cells Transfected with Donor Plasmid.

Mock, untransfected cells; hTp.nori.Con, pT.hTp.nori.Con; mSB, pCMV-mSB (mutated SB transposase); SB, pCMV-SB (SB transposase) (20 X).

respectively. No significant transcriptional activity was seen in human normal primary hepatocytes (hNHeps). These results indicate that the SV40 enhancer increases the activity of telomerase promoter activity in $\mathrm{HCC}$ cell lines, and these are in good agreement with our previous results. ${ }^{8)}$

To confirm long-lasting gene expression using the hTERTp and SV40 enhancer in the SB delivery system, SB vectors with the hTERTp and SV40 enhancer were used. Hep3B cells co-transfected with pT.hTp.Con and active helper plasmid (pCMV-SB) gave rise to about 2-fold higher level of luciferase activity at 5 and $10 \mathrm{~d}$ after transfection, in comparison with a mutant form of the helper plasmid (pCMV-mSB) (Fig. 3).

To test transpositional integration in cultured cells, we transfected a plasmid, pT.hTp.nori.Con, containing a neomycin resistant gene (neo), with helper plasmids into HepG2 cells and selected G418 resistant foci. As Fig. 4 indicates, cells transfected with active helper plasmid (pCMV-SB) form about 50 -fold more $\mathrm{G} 418^{\mathrm{R}}$ foci than with the untranfected mock control or mutant form helper plasmid (pCMV-mSB) (Fig. 4). In Fig. 3, SB transposase increased luciferase activity only 2-fold, although it increased the G418-resistant colonies about 50-fold (Fig. 4). The large difference between luciferase assay and G418-resistance assay might have been due to G418 selection. G418 made the cells where the neo genes were transpositionally integrated by SB transposase thrive more than the non-integrated cells, but there was no selection factor in case shown in Fig. 3, and that perhaps made the difference between the two results.

HCCs and hNHeps were transfected with pT.hTp.HSVtk.Con with active and with inactive helper plasmid with $50 \mu \mathrm{g} / \mathrm{ml}$ of GCV in order to determine the specificity of cancer-directed cell death. Although most of the tumor cells displayed typical cell death, this was not observed in normal human hepatocyte cells after $10 \mathrm{~d}$ of transfection (data not shown).

By Luminescent Cell Viability Assay, pT.hTp.HSVtk.Con with inactive helper plasmid resulted in 33, 39, and $23 \%$ more cell death respectively than the mock control (untransfected but GCV treated) HepG2, Hep3B, and Huh7 cells (Fig. 5). However, pT.hTp.HSV-tk.Con with active helper plasmid resulted in 66, 72, and $40 \%$ more cell death than with mock control cells (Fig. 5). No significant increase in cell death was observed in hNHeps cells (Fig. 5). Similar results were obtained by trypan blue exclusion assay (data not shown).

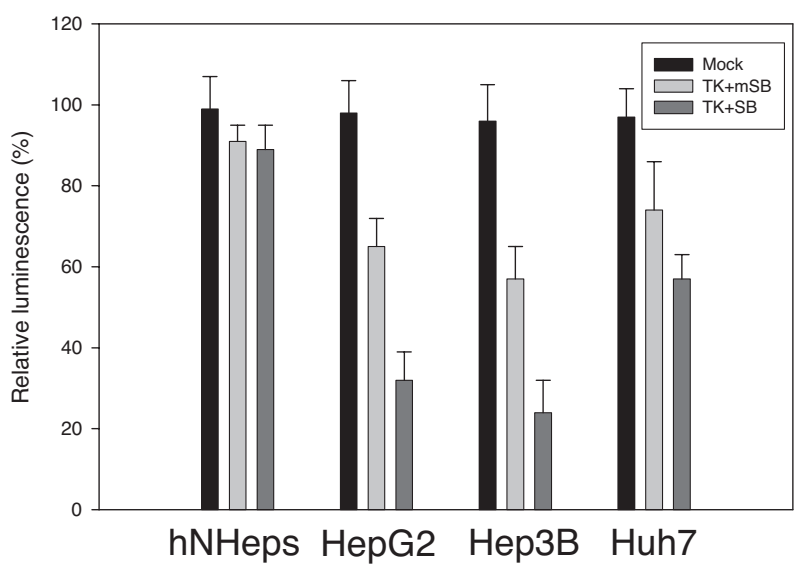

Fig. 5. ATP Cell Viability Assay.

Exposure of HCC cells in vitro to pT.hTp.HSV-tk.Con with active and with inactive helper plasmid + GCV $(50 \mu \mathrm{g} / \mathrm{ml})$ and determination of the number of viable cells by luminescent cell viability assay $10 \mathrm{~d}$ later. Mock, untransfected cells; TK, pT.hTp.HSV-tk.Con; mSB, pCMV-mSB (mutated SB transposase); SB, pCMV-SB (SB transposase); bars, SD.

SB is an ideal delivery system because it lacks many of the drawbacks associated with viral gene delivery. It can provide prolonged transgene expression without eliciting an immunogenic response in the host. There is no risk of recombination and the constructs are easy to make for mass production. It is known that HSV-TK phosphorylates the nontoxic prodrug GCV, which then becomes phosphorylated by endogenous kinases to GCV-triphosphate, causing chain termination and single-strand breaks upon incorporation into the DNA. ${ }^{9)}$ Even when only $10 \%$ of the HSV-TK-expressing tumor cells were inoculated, GCV administration caused tumor regression. ${ }^{10)}$ Prolonged expression of suicide genes by the SB-delivery system might eradicate remaining cancer cells that cannot be treated by anticancer medicine.

In this study we investigated the utility of a SBmediated vector containing a HSV-TK gene under the control of the hTERT promoter, and SV40 enhancer was constructed to target HCCs. This plasmid was constructed and introduced into normal hepatocytes and HCC cell lines. After treatment with GCV, the transfected HCC cells, but not normal hepatocytes, underwent cell death. These results indicate that SBmediated suicide gene delivery might be a useful method of suppressing tumor growth in targeted cancer gene therapy. 


\section{Acknowledgments}

We thank Dr. Mark A. Kay for providing pT-MCS, pTnori, pCMV-SB, and pCMV-mSB. This work was supported by K08 DK05880 (to E.R.O.) and the Korea Science and Engineering Foundation (KOSEF) through the Tumor Immunity Medical Research Center (TIMRC) at Seoul National University College of Medicine (to C.W.K.).

\section{References}

1) Yant, S. R., Meuse, L., Chiu, W., Ivics, Z., Izsvak, Z., and Kay, M. A., Somatic integration and long-term transgene expression in normal and haemophilic mice using a DNA transposon system. Nat. Genet., 25, 35-41 (2000).

2) Song, J. S., Murase, N., Demetris, A. J., Michalopoulos, G. K., and Ochoa, E. R., Protection from acute cellular injury using Sleeping Beauty mediated telomerase gene transfer. Biochem. Biophys. Res. Commun., 363, 253-256 (2007).

3) Ohlfest, J. R., Demorest, Z. L., Motooka, Y., Vengco, I., Oh, S., Chen, E., Scappaticci, F. A., Saplis, R. J., Ekker, S. C., Low, W. C., Freese, A.B., and Largaespada, D. A., Combinatorial antiangiogenic gene therapy by nonviral gene transfer using the Sleeping Beauty transposon causes tumor regression and improves survival in mice bearing intracranial human glioblastoma. Mol. Ther., 12, 778-788 (2005).

4) Ohlfest, J. R., Lobitz, P. D., Perkinson, S. G., and Largaespada,
D. A., Integration and long-term expression in xenografted human glioblastoma cells using a plasmid-based transposon system. Mol. Ther., 10, 260-268 (2004).

5) Izumi, H. P., Louann, C., Cynthia, A., and Carl, B., Cloning and characterization of the promoter region of the human telomerase reverse transcriptase gene. Cancer Res., 59, 826-830 (1999).

6) Masahiro, T., Satoru, K., Taro, K., Hisao, H., Jun, T., Masuo, Y., and Masaki, I., Cloning of human telomerase catalytic subunit (hTERT) gene promoter and identification of proximal core promoter sequences essential for transcriptional activation in immortalized and cancer cells. Cancer Res., 59, 551-557 (1999).

7) Youssef, N., Paradis, V., Ferlicot, S., and Bedossa, P., In situ detection of telomerase enzymatic activity in human hepatocellular carcinogenesis. J. Pathol., 194, 459-465 (2001).

8) Song, J. S., Activity of the human telomerase catalytic subunit (hTERT) gene promoter could be increased by the SV40 enhancer. Biosci. Biotechnol. Biochem., 68, 1634-1639 (2004).

9) Reid, R., Mar, E. C., Huang, E. S., and Topal, M. D., Insertion and extension of acyclic, dideoxy, and ara nucleotides by herpesviridae, human alpha and human beta polymerases: a unique inhibition mechanism for 9-(1,3-dihydroxy-2-propoxymethyl)guanine triphosphate. J. Biol. Chem., 263, 3898-3904 (1988).

10) Freeman, S. M., Abboud, C. N., Whartenby, K. A., Packman, C. H., Koeplin, D. S., Moolten, F. L., and Abraham, G. N., The "bystander effect": tumor regression when a fraction of the tumor mass is genetically modified. Cancer Res., 53, 5274-5283 (1993). 